\title{
GAS DYNAMICS IN THE GALACTIC CENTRE: GRAVITY VS. MAGNETISM
}

\author{
Ralph E. Pudritz \\ Dept. of Physics, McMaster University, Hamilton, ON L8S 4M1
}

\section{INTRODUCTION}

This contribution is a summary of an impromptu and extremely interesting discussion on the nature of the forces acting on gas in the galactic centre. The origins of the gas motions in the central few parsecs are crucial for clarifying many important problems about galactic nuclei. Some of the many gas dynamics issues that were raised include: 1 . are there explosive events in the galactic centre which produce outward moving gas clouds (see Heyvaerts, these proceedings)? 2. are the streamers of gas due to the tidal disruption of molecular clouds that stray in too deep in the galactic centre's tidal field (see Serabyn, these proceedings)? 3 . is there circular gas motion which would be important in constraining the nature of the mass distribution inside scales of a few pc (see review by Townes, these proceedings)?

The discussion centered on gas dynamics in the molecular torus and ionized gas streamers on the 2-5 pc scale. Because reliable measurements of the magnetic field strength in the galactic centre are not yet available, several of the key points of contention will not be finally settled until the data are in. Be this as it may, the discussion boiled down to the issue of whether gravity or magnetic fields were responsible for the structures and morphology on this scale. Accordingly I will label the arguments of the two relevant camps by $\mathbf{G}$ (for gravitational fields as the predominant shaper of things) and $\mathbf{M}$ (for magnetic fields as the important agents).

\section{IONIZED GAS STREAMERS}

G: Tidally Disrupted Clouds;

Lacy (see also his contribution, these proceedings) argued that gravity could easily disrupt and stretch out molecular clouds into infalling, 1-D gas streamers with little effort on the 2 pc. scale. In particular, the Northern Arm and its possible extension into the east-west bar are consistent with gravitational forces. Thus, a cloud with a significant but not dominant magnetic field will be stretched out and laterally compressed by the tidal forces which are dismembering it, readily producing the structure we apparently see.

M: Magnetic Loops and Strong Cloud Fields;

Heyvaerts opened the counter argument to Lacy's point. The magnetic field in the tidally disrupted streamer is not necessarily strengthened up to equipartition 
with the full gravitational potential energy, but would be a factor of $(\Delta r / r)^{2}$ smaller than the gravitational energy density (where $\Delta r$ is the width of the streamer). Thus, if the field were not originally quite strong in the cloud, disruption by tides on the observed scales by itself won't get it up to the value required by the observations. Yet another issue here is the direction of the polarization vectors along this northern arm. If the magnetic field in this filament were produced by stretching, it would be oriented parallel to the filament and the polarization vector would be oriented perpendicular to the filament for dust seen in emission. This is what the polarization observations at 10 and $100 \mu \mathrm{m}$ show (see review by Aitken, these proceedings), but there is another explanation of this geometry as well (see below).

A puzzling feature about the strictly gravitational interpretation of the run of gas velocities down the northern arm and into the east-west bar is the presence of a clear break at the intersection of these two features. In the MHD model, the eastwest bar is a piece of expanding magnetic loop which continues out to larger scales. The interaction point is where reconnection of loop with torus fields occurs, and all kinds of velocity anamolies are to be expected (see Heyvaerts, Norman and Pudritz 1988, henceforth HNP). The discussion raised the question of why the interaction point is so far away from the HCN torus (Genzel, these proceedings). A possible explanation of this fact is that the HCN only traces the densest part of the disk. The $50 \mu \mathrm{m}$ dust emission data shown by Werner (these proceedings) makes it likely that lower density but still cool material is to be found in the immediate vicinity of the crossing of the east west bar and the northern arm.

\section{POLARIZATION VECTORS}

G: The polarization vectors along the molecular torus are somewhat puzzling if a purely magnetic model is accurate. Townes raised the question of how do we understand the polarization vectors as measured by Hildebrand and collaborators, which lie perpendicular to the torus plane. The data suggest that the magnetic field lies in the plane of the molecular torus and may continue out in the lower density regions of an extended disk. An additional point to keep in mind is that filamentary features which fill the Sgr A West region appear to be poloidal (oriented perpendicular to the plane of the $2-5$ pc molecular torus or disk), and may have their roots in the molecular torus. The complexity of the polarization data apparently make a simple MHD theory rather difficult.

M: There are several ideas germane to the previous questions. One important point is that the shearing molecular disk will certainly have an associated toroidal field component that will lie in the plane of the disk. One should picture the molecular disk and the associated filament system comprising Sgr A, as a disk/magnetosphere or corona (HNP; Heyvaerts, these proceedings). Field lines while perpendicular to the disk in the "corona", will be sheared on entering the torus. This effect inevitably gives rise to a toroidal field component which explains Hildebrand's 100 $\mu \mathrm{m}$ observations (see eg. Aitken's contribution, these proceedings). Rosner noted that if a turbulent dynamo were operative in the galactic halo, it would be possible to generate a dipolar magnetic field. This would account for the apparently poloidal field (ie. perpendicular to galactic plane) suggested by the polarimetry on larger scales than that of the torus.

It is worth keeping in mind that in other parts of the galactic interstellar medium, the magnetic field is observed to take on a variety of orientations with respect to the dense gas. As an example, filaments of molecular gas are seen to have 
magnetic field oriented generally parallel to the long axis of the filament. In Taurus however, one sees ordered field perpendicular to the dense filaments (see eg. Strom et al 1988). This is much the same magnetic geometry as is seen in the molecular torus. A further cautionary note on the interpretation of polarization data was provided by Ekers, during the conference. Even a rather simple source geometry can produce apparently chaotic looking polarization maps. A simple example of this is a Faraday screen with a gradient across it.

\section{IONIZATION AGENTS}

\section{G: Photo-ionization;}

The heating of the ionized gas streamers on these 2-pc scales is still somewhat of a mystery. Townes, in his review talk, discussed the relative merits of shock heating and photo-dissociation. The [CII] line emission observed in the molecular torus is quite intense and is difficult to attribute to the effects of shock heating. The suggested solution is that photoionization could possibly reproduce the correct line intensities. Genzel (these proceedings ) noted that the large amount of unusually warm gas could be produced by either cloud-cloud collisions or photoelectric heating by UV radiation generated by a cluster of $O$ and $B$ stars at the center.

\section{M: Alfvenic Heating;}

The idea that photoionization is driving the ionized gas physics is not without its own problems since the galactic centre stellar population must be truncated at O7, in order to avoid a too high ionizing flux. HNP suggested that the molecular torus is undergoing strong MHD shocks due to the presence of rapidly expanding magnetic loops. This process produces finite amplitude Alfven waves in the strongly magnetized torus. These waves steepen and dissipate their energy ultimately heating the gas to the observed temperatures. While the energetics and gas temperatures are consistent with the observations, it remains to be seen whether detailed predictions for the various line intensities can be calculated.

Thus, another way of producing ionized gas emission on the inner edge of the molecular torus is to heat the gas by the damping of Alfvenic surface waves which have been excited by the collision of an expanding magnetic loop with the magnetized molecular torus.

\section{TORUS DYNAMICS}

A large part of the discussion centered on the question of how the torus is able to retain its integrity over a time scale of even $10^{6}$ years. The issue here is that the individual clumps in the torus ought to spiral into the center on time scales of order $10^{5}$ years or so. Genzel stressed that this was an important problem for any theoretical model.

\section{G: Purely Gravitational Processes;}

It is difficult to prescribe exactly what is driving the instabilities producing the clumps in a model of molecular clouds in which magnetic fields do not play a role. The rapid clump-clump collisions that occur in the torus will result in rapid loss of angular momentum from the torus clumps. This leads to short torus lifetimes in the absence of other cloud "bonding" agents. It should be emphasized that molecular clouds in the galactic center must have faster dynamical time scales since they have such high surface densities compared to garden variety clouds in the solar vicinity. This point was made by Stark (these proceedings) when he 
emphasized that in the tidal field of the galactic center, clouds must have densities $>10^{4} \mathrm{~cm}^{-3}$ and velocity dispersions of $>30 \mathrm{~km} \mathrm{~s}^{-1}$ in order to be self gravitating. Thus the dynamical time scale on 2 -pc torus scales is $2 \mathrm{pc} / 30 \mathrm{~km} \mathrm{~s}^{-1} \simeq 10^{5} \mathrm{yr}$.

The question of the origin of the cores in the molecular torus however, is not too different (other than the different values for virial velocities and cloud densities) than that faced in understanding clouds anywhere in the interstellar medium.

M: Hydromagnetic Waves in Molecular Clouds:

It is precisely the issue of torus dynamics that magnetic field effects elucidate. It is well known now that a wide variety of galactic molecular clouds have magnetic field strengths comparable to gravitational energy density (e.g., Myers and Goodman 1988). This implies that molecular clouds can be supported against collapse by the gas motions induced by hydromagnetic waves in the cloud. There are several mechanisms available for the formation of cores in magnetized clouds. The presence of compressive hydromagnetic modes (so called fast magnetosonic modes) closely related to Alfven waves will actually produce density fluctuations. These perturbations act as seeds for the eventual growth of a spectrum of clumps, with properties similar to that of cores. This simple idea has been demonstrated in preliminary N-body simulations (Pudritz and Carlberg 1988), and is currently being actively pursued by us. Cores are vibrated back and forth around "home" positions in this wave picture so that angular momentum loss which occurs during the collision of any two clumps is shared up with the whole collection, thereby reducing the infall rate of any one clump.

\section{SUMMARY}

Many of the physical processes thought to be important in local clouds probably play a central role in the molecular clouds in the galactic centre. Some of the questions raised in the discussion will be resolved when actual measurements of magnetic field strengths are obtained. The observation of very strong fields in local clouds by direct observation of Zeeman splitting of $\mathrm{OH}$ absorption lines is a very hopeful possibility in this regard.

It is a great pleasure to thank Mark Morris for assembling and orchestrating such a stimulating and timely meeting on this most important topic. This work was supported by NSERC of Canada.

\section{REFERENCES}

Arons J., and Max, C.E. 1975, Ap. J. (Letters), 196, 177.

Heyvaerts J., Norman C.A., and Pudritz, R.E. 1988 (HNP), Ap. J., 330, 718.

Myers, P.C., and Goodman, A. 1988, Ap. J. (Letters), 326, L31.

Pudritz, R.E., and Carlberg, R.G. 1988, in

Molecular Clouds in the Milky Way and External Galaxies,

R. Dickman, R. Snell and J. Young eds. (Springer-Verlag; New York), in press.

Strom, S.E., Strom, K.M., and Edwards, S. 1988, in

Galactic and Extragalactic Star Formation, R.E. Pudritz, and M. Fich eds., (D. Reidel; Dordrecht), p. 53. 